\title{
Article \\ The Magnetic Hooke-Newton Transmutation in Momentum Space
}

\author{
De-Hone Lin
}

check for

updates

Citation: Lin, D.-H. The Magnetic Hooke-Newton Transmutation in Momentum Space. Symmetry 2021, 13, 608. https://doi.org/10.3390/sym 13040608

Academic Editor: Roberto Passante

Received: 28 January 2021

Accepted: 29 March 2021

Published: 6 April 2021

Publisher's Note: MDPI stays neutral with regard to jurisdictional claims in published maps and institutional affiliations.

Copyright: (C) 2021 by the author. Licensee MDPI, Basel, Switzerland. This article is an open access article distributed under the terms and conditions of the Creative Commons Attribution (CC BY) license (https:// creativecommons.org/licenses/by/ $4.0 /)$.
Department of Physics, National Sun Yat-sen University, Kaohsiung 80424, Taiwan; dhlin@mail.nsysu.edu.tw

\begin{abstract}
The magnetic Hooke-Newton transmutation, which emerges from the transformation design of the quadratic conformal mapping for the system of charged particles moving in the uniform magnetic field, is investigated in the momentum space. It is shown that there are two ways to turn the linear interaction force of the system into the inverse square interaction. The first one, which involves simply applying the mapping to the system, has the spectrum with the Landau levels of even angular momentum quantum number. The second one considers the geometric structure of the mapping as an effective potential which leads us to the transmuted Coulomb system with the novel quantum spectrum. The wave functions of momentum for the bound and scattering states of the transmutation system are given. It is also shown that the effective potential due to the geometric structure can be generalized to a general 2D surface, and the Schrödinger equation of a particle moving on the surface while under the action of the potential can be solved by the form-invariant Schrödinger equation of the free particle. The solution of a particle moving on the hyperbolic surface under the potential is given with the conclusion. The presentation manifests the transformation design of the quantum state, depending mainly on the geometric structure of the representation space, not on the action of the specific potential field. This characteristic makes it possible for us to use the geometric structure of different representation spaces to explore some novel behaviors of quantum particles.
\end{abstract}

Keywords: momentum representation; transformation design of quantum state; conformal mapping; magnetic Hooke-Newton transmutation

\section{Introduction}

The transformation design method (TDM) was introduced in optics [1,2], and has been extensively applied to the diverse wave phenomena (e.g., [3]). Through the reinterpretation of the geometric structure of the transformation as the material properties, the method enables novel devices such as invisibility cloaks (e.g., [4]). Recently, researchers have been interested in the applications of transformation design to the steering of matter waves (e.g., $[5,6])$. Due to the interaction nature of the quantum particle with external fields, the concept of the transformation design in quantum physics could exhibit more fertile content such as leading us to reveal new quantization systems (e.g., [7]) and more general monopole fields [8].

In quantum mechanics, one of the interesting contents is the representation theory that allows us to perceive quantum phenomena from different viewpoints, and may afford an easier way to comprehend a quantum system. It is acknowledged that the momentum representation has its own virtue in offering the visible image of the quantum wave functions (e.g., [9]) and the understanding of the quantum condensation phenomena. So far, few of the transformation designs have been discussed through the momentum space ( $p$ space) [10]. Thus, it would be worth exploring the topic in the p-space that may enlarge the application of the representation theory. In this paper, we investigate the magnetic HookeNewton transmutation in the p-space through reinterpreting the geometric structure of the quadratic conformal mapping as an effective interacting potential. The exploration offers an alternative viewpoint to inspect the system while confirming the effectiveness of the TDM in the representation spaces other than the coordinate space. 
Our discussion is arranged as follows: In Section 2, the quadratic conformal mapping is applied to the momentum representation of the Schrödinger equation for the charge particle coupling to a uniform magnetic field, and obtains the equation of a transmuted Coulomb system. It is shown that the transmutation system only has the Landau levels of even angular momentum quantum number. In Section 3, an additional interaction due to an effective potential defined by the geometric structure of the conformal mapping is considered in the equation of the Landau level system. It is then shown that the mapping turns the new equation into the transmuted Coulomb system with the novel quantum spectrum discovered in the recent investigation of the transformation design of quantum states [11]. The momentum wave functions of bounded and scattering states are obtained. Our conclusion is presented in Section 4. Finally, in the Appendix A, the definition of the effective potential is generalized to a general 2D surface, and we explain how to construct the form-invariant Schrödinger equation on the surface with the potential, and solve the quantum problem of a particle interacting with the potential.

\section{The Quadratic Conformal Image of the Equation for a Charge in the Uniform Magnetic Field in Momentum Space}

In the coordinate space, the Hamilton operator of a charged particle coupling to a magnetic field is known to be

$$
\hat{H}=\frac{1}{2 M}\left(\hat{\mathbf{P}}-\frac{q}{c} \mathbf{A}\right)^{2}
$$

For a uniform magnetic field with strength $B$ and pointing along the $z$-axis, the vector potential can be chosen as

$$
\mathbf{A}=\frac{B}{2}\left(-y \hat{e}_{x}+x \hat{e}_{y}\right) .
$$

In the following content, we shall restrict our discussion to the $2 \mathrm{D}$ plane since along the $z$-axis the particle just performs free motion. The substitution of (2) in (1) gives the expression of the operator

$$
\hat{H}=\frac{1}{2 M} \hat{\mathbf{P}}^{2}+\omega\left(y \hat{p}_{x}-x \hat{p}_{y}\right)+\frac{M \omega^{2} \rho^{2}}{2}
$$

where $\omega=q B / 2 M c, \rho^{2}=x^{2}+y^{2}$, and $\hat{\mathbf{P}}=\left(\hat{p}_{x}, \hat{p}_{y}\right)$ in the components. The quantum states $\Psi(\mathbf{x})$ corresponding to the operator are determined by the Schrödinger equation

$$
\left[\frac{1}{2 M} \hat{\mathbf{P}}^{2}+\omega\left(y \hat{p}_{x}-x \hat{p}_{y}\right)+\frac{M \omega^{2} \rho^{2}}{2}\right] \Psi(\mathbf{x})=E \Psi(\mathbf{x}) .
$$

The momentum representation of the states $\Psi(\mathbf{p})$ is associated with $\Psi(\mathbf{x})$ by the Fourier transformation

$$
\Psi(\mathbf{x})=\frac{1}{(2 \pi \hbar)^{2}} \int \Psi\left(\mathbf{p}_{1}\right) e^{i \mathbf{p}_{1} \cdot \mathbf{x} / \hbar} d \mathbf{p}_{1} .
$$

By replacing the wave function in (4) by the integral representation, the Schrödinger equation is shown as

$$
\int d \mathbf{p}_{1}\left\{\frac{\mathbf{p}_{1}^{2}}{2 M}+\omega\left(p_{1 x} y-p_{1 y} x\right)+\frac{M \omega^{2} \rho^{2}}{2}\right\} \Psi\left(\mathbf{p}_{1}\right) e^{i \mathbf{p}_{1} \cdot \mathbf{x} / \hbar}=E \int d \mathbf{p}_{1} \Psi\left(\mathbf{p}_{1}\right) e^{i \mathbf{p}_{1} \cdot \mathbf{x} / \hbar}
$$

Here $p_{1 x}$ stands for the first component of $\mathbf{p}_{1}$, i.e., $\mathbf{p}_{1}=\left(p_{1 x}, p_{1 y}\right)$. Multiply both sides of the equation by $e^{-i \mathbf{p}_{2} \cdot \mathbf{x} / \hbar}$ from the right, and note that 


$$
\left[\omega\left(p_{1 x} y-p_{1 y} x\right)+\frac{M \omega^{2} \rho^{2}}{2}\right] e^{i\left(\mathbf{p}_{1}-\mathbf{p}_{2}\right) \cdot \mathbf{x} / \hbar}=\left\{\omega\left[p_{1 x}\left(i \hbar \frac{\partial}{\partial p_{2 y}}\right)-p_{1 y}\left(i \hbar \frac{\partial}{\partial p_{2 x}}\right)\right]+\frac{M \omega^{2}}{2}\left(i \hbar \nabla_{\mathbf{p}_{2}}\right)^{2}\right\} e^{i\left(\mathbf{p}_{1}-\mathbf{p}_{2}\right) \cdot \mathbf{x} / \hbar}
$$

Then, taking the integration over the coordinate space $\int d \mathbf{x}$, and employing the welcome formula of the Dirac's function,

$$
\int d \mathbf{x} e^{i\left(\mathbf{p}_{1}-\mathbf{p}_{2}\right) \cdot \mathbf{x} / \hbar}=(2 \pi \hbar)^{2} \delta\left(\mathbf{p}_{1}-\mathbf{p}_{2}\right),
$$

we get the momentum representation of Equation (4),

$$
\left\{\frac{-\hbar^{2}}{2 A} \nabla_{\mathbf{p}}^{2}+i \hbar \omega\left(p_{x} \frac{\partial}{\partial p_{y}}-p_{y} \frac{\partial}{\partial p_{x}}\right)\right\} \Psi(\mathbf{p})=\left(E-\frac{\mathbf{p}^{2}}{2 M}\right) \Psi(\mathbf{p}),
$$

where $\nabla_{\mathbf{p}}^{2}=\left(\partial / \partial p_{x}\right)^{2}+\left(\partial / \partial p_{y}\right)^{2}$, and the parameter $A=1 /\left(M \omega^{2}\right)$. The kinetic energy operator in the $\mathrm{p}$-space becomes the potential function $\mathrm{p}^{2} / 2 M$ on the right hand side. Because of the system with cylindrical symmetry, it is convenient to discuss the equation in the polar coordinates $(p, \varphi)$ defined by $p^{2}=\mathbf{p}^{2}=p_{x}^{2}+p_{y}^{2}$ and $\varphi=\tan ^{-1} p_{y} / p_{x}$. The relationship between the Cartesian coordinates $\left(p_{x}, p_{y}\right)$ and the polar coordinates is given by $p_{x}=p \cos \varphi, p_{y}=p \sin \varphi$, and the derivatives between these two coordinate systems are associated with

$$
\frac{\partial}{\partial p_{x}}=\cos \varphi \frac{\partial}{\partial p}-\frac{\sin \varphi}{p} \frac{\partial}{\partial \varphi}
$$

and

$$
\frac{\partial}{\partial p_{y}}=\sin \varphi \frac{\partial}{\partial p}+\frac{\cos \varphi}{p} \frac{\partial}{\partial \varphi}
$$

Using these expressions, it is easy to show that Equation (9) has the polar representation

$$
\left\{\frac{-\hbar^{2}}{2 A}\left[\frac{\partial^{2}}{\partial p^{2}}+\frac{1}{p} \frac{\partial}{\partial p}+\frac{1}{p^{2}} \frac{\partial^{2}}{\partial \varphi^{2}}\right]+i \hbar \omega \frac{\partial}{\partial \varphi}\right\} \Psi(\mathbf{p})=\left(E-\frac{p^{2}}{2 M}\right) \Psi(\mathbf{p}) .
$$

Although the solution of the equation is a well-known result, let us spend a little space to state it for comparison with the later works. By inserting the ansatz $\Psi(\mathbf{p})=R(p) e^{i m \varphi}$ into the equation, the radial momentum wave is found to satisfy the equation

$$
\left[\left(\frac{d^{2}}{d p^{2}}+\frac{1}{p} \frac{d}{d p}-\frac{m^{2}}{p^{2}}\right)+k^{2}-\left(\frac{p}{M \hbar \omega}\right)^{2}\right] R(p)=0,
$$

with the constant $k^{2} \equiv 2 A(E+m \hbar \omega) / \hbar^{2}$ for short. The asymptotic behaviors at $p \rightarrow 0$ and $p \rightarrow \infty$ of the equation require that the radial wave must be the product form

$$
R(p)=p^{|m|} e^{-\left(p^{2} / 2 M \hbar \omega\right)} u(p)
$$

Substitution of the form in (13) gives the equation for the function $u$,

$$
\left[\xi \frac{d^{2}}{d \xi^{2}}+(|m|+1-\xi) \frac{d}{d \xi}-\left(\frac{|m|+1}{2}-\frac{M \hbar \omega}{4} k^{2}\right)\right] u=0,
$$

where the variable $\xi=p^{2} /(M \hbar \omega)$. This is the standard form of the confluent hypergeometric equation (see, e.g., p. 268, [12]). Its analytic solution around $\xi \approx 0$ is the confluent hypergeometric function $F(\eta, \gamma ; \xi)$ with the parameters 


$$
\eta=\frac{|m|+1}{2}-\frac{M \hbar \omega}{4} k^{2}, \text { and } \gamma=|m|+1 .
$$

The condition of the bound states requires

$$
\eta=-n_{p}, \quad n_{p}=0,1,2, \cdots .
$$

Thus, the energy levels hidden in $k$ read

$$
E=\hbar \omega\left(2 n_{p}+|m|-m+1\right) \text {, with } m=0, \pm 1, \pm 2, \cdots .
$$

The quantization values of $m$ are determined by the single-valuedness condition of the wave function.

What we are interested in is the transmutation of (12) due to the quadratic conformal mapping

$$
\bar{p}=\frac{p^{2}}{p_{0}}, \text { and } \bar{\varphi}=2 \varphi,
$$

where $p_{0}$ is a constant with the units of momentum used to balance the units of both sides. It will be determined in the next section. We shall see that the mapping would associate the Landau-level system with the Coulomb system in the p-space. Change the variables with the mapping. The derivatives in (12) turn into

$$
\frac{\partial}{\partial p}=\bar{p}^{\prime} \frac{\partial}{\partial \bar{p}^{\prime}}, \frac{\partial^{2}}{\partial p^{2}}=\bar{p}^{\prime \prime} \frac{\partial}{\partial \bar{p}}+\left(\bar{p}^{\prime}\right)^{2} \frac{\partial^{2}}{\partial \bar{p}^{2}},
$$

and

$$
\frac{\partial}{\partial \varphi}=\bar{\varphi}^{\prime} \frac{\partial}{\partial \varphi}, \frac{\partial^{2}}{\partial \varphi^{2}}=\left(\bar{\varphi}^{\prime}\right)^{2} \frac{\partial^{2}}{\partial \bar{\varphi}^{2}},
$$

where $\bar{p}^{\prime}=d \bar{p} / d p$ and $\bar{\varphi}^{\prime}=d \bar{\varphi} / d \varphi$, which make (12) become

$$
\left\{\frac{-\hbar^{2}}{2 A}\left[\left(4 \frac{\bar{p}}{p_{0}}\right) \frac{\partial^{2}}{\partial \bar{p}^{2}}+\left(\frac{4}{p_{0}}\right) \frac{\partial}{\partial \bar{p}}+\left(\frac{4}{p_{0} \bar{p}}\right) \frac{\partial^{2}}{\partial \bar{\varphi}^{2}}\right]+2 i \hbar \omega \frac{\partial}{\partial \bar{\varphi}}\right\} \Psi(\overline{\mathbf{p}})=\left(E-\frac{p_{0} \bar{p}}{2 M}\right) \Psi(\overline{\mathbf{p}}) .
$$

By the decomposition $\Psi(\overline{\mathbf{p}})=R(\bar{p}) e^{i \mu \bar{\varphi}}$ of the wave function, the equation is transformed into

$$
\left[\left(\frac{d^{2}}{d \bar{p}^{2}}+\frac{1}{\bar{p}} \frac{d}{d \bar{p}}-\frac{\mu^{2}}{\bar{p}^{2}}\right)+\frac{\alpha}{\bar{p}}\right] R(\bar{p})=E_{0} R(\bar{p}),
$$

where

$$
\alpha=\left[\frac{p_{0} \mu}{M \hbar \omega}+\frac{p_{0} E}{2 M(\hbar \omega)^{2}}\right] \text {, and } E_{0}=\left(\frac{p_{0}}{2 M \hbar \omega}\right)^{2} .
$$

It is noticed that Equation (23) is the radial equation of the Coulomb system in variable $\bar{p}$. However, $E_{0}$ on the right hand side of (23) is not an eigenenergy to be evaluated. It is just a constant composed of the proper constants of the system. One can regard $E_{0}$ as the eigenvalue, and we can solve the equation to find the genuine eigenenergy $E$ hidden in the coupling constant $\alpha$ now. With the asymptotic solutions of the equation at the near and far zones of $p$, the radial wave can be written in the form

$$
R(\bar{p}) \sim \bar{p}^{|\mu|} e^{-\beta \bar{p}} u(\bar{p}), \text { with } \beta=\sqrt{E_{0}} .
$$

The replacement of the product form in (23) gives the equation satisfied by $u(\bar{p})$

$$
\left[\zeta \frac{d^{2}}{d \zeta^{2}}+(2|\mu|+1-\zeta) \frac{d}{d \zeta}-\left(|\mu|+\frac{1}{2}-\frac{\alpha}{2 \beta}\right)\right] u=0,
$$

where $\zeta=2 \beta \bar{p}$ was defined. We see the confluent hypergeometric equation again, and its solution is the confluent hypergeometric function $F(\eta, \gamma ; \zeta)$ with the parameters 


$$
\eta=|\mu|+\frac{1}{2}-\frac{\alpha}{2 \beta}, \text { and } \gamma=2|\mu|+1
$$

The bound state condition requires

$$
\eta=-n_{p}, \quad n_{p}=0,1,2, \cdots
$$

With the parameters in (24), the energy levels are found to have the expression

$$
E=\hbar \omega\left(2 n_{p}+2|\mu|-2 \mu+1\right) .
$$

The value of the constant $\mu$ is determined by the single-valuedness condition $e^{i \mu \bar{\varphi}}=e^{i \mu(\bar{\varphi}+2 \pi)}$ of the wave function of the transmuted system which shows

$$
\mu=m, m=0, \pm 1, \pm 2, \cdots .
$$

It follows that the energy levels (29) with the single-valuedness wave function are

$$
E=\hbar \omega\left(2 n_{p}+2|m|-2 m+1\right) .
$$

Therefore, the conformal mapping turns the Landau levels of odd angular momentum quantum number into even number. It does not alter the physical processes associated with the transition among even quantum numbers. However, the processes with the levels of odd angular momentum are cancelled. The transmutation between the linear force law and the inverse square force law was first pointed out by Issac Newton in his book (Cor. III, Prop. VII, [13], and, e.g., [14-16]). The magnetic version of the transmutation with a novel spectrum was discussed in the recent works $[11,17,18]$.

Remark 1. In References $[11,17,18]$, the quantum number $\mu$ of the transmutation system was taken as $\mu=m / 2$ which leads to the solution of the Schrödinger equation in the target space being two-value function, thus violating the single-valuedness condition of the wave function. The single-valuedness solution can be obtained by replacing the quantum number $m$ by $2 m$ therein. A similar mistake also appeared in [8]. The author very much appreciates the referee for his comments on this point.

\section{The Magnetic Hooke-Newton Transmutation Due to the Action of an Effective Potential}

What is more interesting in the transmutation caused by the quadratic mapping is additionally considering the geometric structure of the mapping as an effective potential which could turn the Schrödinger equation with the potential in the p-space into the forminvariant equation of the free particle (see Appendix A for details). The potential due to the mapping under consideration is defined by

$$
U=[1-\text { conformal factor }] E=\left[1-\left(\bar{p}^{\prime}\right)^{2}\right] E=\left(1-\frac{4 \bar{p}}{p_{0}}\right) E .
$$

Add the potential to Equation (23). It follows that

$$
\begin{gathered}
{\left[\left(\frac{d^{2}}{d \bar{p}^{2}}+\frac{1}{\bar{p}} \frac{d}{d \bar{p}}-\frac{\mu^{2}}{\bar{p}^{2}}\right)+\frac{\left(p_{0} \mu / M \hbar \omega\right)}{\bar{p}}\right] R(\bar{p})} \\
=\left[-\frac{p_{0}}{2 M(\hbar \omega)^{2}} \frac{(E-U)}{\bar{p}}+E_{0}\right] R(\bar{p}) .
\end{gathered}
$$

The first term on the right hand side simply gives $-2 A E / \hbar^{2}$, and the equation becomes

$$
\left[\left(\frac{d^{2}}{d \bar{p}^{2}}+\frac{1}{\bar{p}} \frac{d}{d \bar{p}}-\frac{\mu^{2}}{\bar{p}^{2}}\right)+\frac{\left(p_{0} \mu / M \hbar \omega\right)}{\bar{p}}\right] R(\bar{p})=-\bar{E} R(\bar{p}),
$$


where

$$
\bar{E}=\frac{2 A}{\hbar^{2}}\left[E-\frac{\left(p_{0} / 2\right)^{2}}{2 M}\right] .
$$

Equation (34) is the radial equation for a Coulomb system in variable $\bar{p}$. In contrast to (23), the right hand side of the transmuted system has the eigenvalue $\bar{E}$, not just a constant. The physical energy level $E$ can be extracted from $\bar{E}$ as soon as $\bar{E}$ is known. One of the interesting features of the system is that the interaction, attractive or repulsive, is determined by both the signatures of the angular momentum and the charge shown in the current coupling:

$$
\alpha=\frac{p_{0} \mu}{M \hbar \omega} .
$$

Since $\mu=m, m=0, \pm 1, \pm 2, \cdots$, and $\omega=q B / 2 M c$, it has the conditions

$$
\left\{\begin{array}{l}
m>0, \text { and } q>0, \text { or } \\
m<0 \text {, and } q<0
\end{array}\right.
$$

for bound states, and

$$
\left\{\begin{array}{l}
m>0, \text { and } q<0, \text { or } \\
m<0, \text { and } q>0,
\end{array}\right.
$$

for scattering states, i.e., for a bound state, the electron (anti-electron) can only move counterclockwise (clockwise) if the direction of the angular momentum $m>0$ is defined as counterclockwise. This observation is consistent with the discussion of the system in the coordinate space [11].

\subsection{Bound State}

Using the asymptotic forms of Equation (34) at $\bar{p} \rightarrow 0$ and $\bar{p} \rightarrow \infty$, one can put the radial wave in the form

$$
R(\bar{p})=\bar{p}^{|\mu|} e^{-\beta \bar{p}} u(\bar{p}),
$$

where $\beta=\sqrt{-\bar{E}}$ with $\bar{E}<0$. Substitution in (34) shows that $u(\bar{p})$ satisfies

$$
\left[\xi \frac{d^{2}}{d \xi^{2}}+(2|\mu|+1-\xi) \frac{d}{d \xi}-\left(|\mu|+\frac{1}{2}-\frac{\alpha}{2 \beta}\right)\right] u=0,
$$

where $\xi=2 \beta \bar{p}$. We have the confluent hypergeometric equation. Its solution is the confluent hypergeometric function $F(|\mu|+1 / 2-\alpha / 2 \beta, 2|\mu|+1 ; \xi)$. Bound states are determined by the quantization condition

$$
|\mu|+\frac{1}{2}-\frac{\alpha}{2 \beta}=-n_{p}, \quad n_{p}=0,1,2, \cdots .
$$

It gives the finite polynomial solution of the bound state wave function. The energy levels are then found to be

$$
E=\frac{\left(p_{0} / 2\right)^{2}}{2 M}\left[1-\frac{\mu^{2}}{\left(n_{p}+|\mu|+1 / 2\right)^{2}}\right] .
$$

It is the place to determine $p_{0}$. The spectrum was recently obtained in the coordinate space and has the definite expression ([11], and Equation (37) in [17])

$$
E=\frac{M\left(\omega_{c} / 2\right)^{2}\left(\rho_{0} / 2\right)^{2}}{2}\left[1-\frac{m^{2}}{\left(n_{p}+|m|+1 / 2\right)^{2}}\right]
$$

with $\omega_{c}=q B / M c$ and the characteristic length of the system $\rho_{0}=\sqrt{4 \hbar /\left(|m| M \omega_{c}\right)}$. A comparison of these two equations shows that 


$$
p_{0}=\sqrt{\frac{2 M \hbar \omega}{|m|}} .
$$

This can be regarded as the characteristic length of the system in the p-space. The length depends on the quantum number $m$ of the angular momentum. This is due to the fact that the Coulomb interaction in (34) depends on $m$. Thus, it is better to consider different $m$ corresponding to the independent system in the transmutation. Without any indefinite coefficient, the energy levels have the explicit expression

$$
E=\frac{\hbar \omega}{4|m|}\left[1-\frac{m^{2}}{\left(n_{p}+|m|+1 / 2\right)^{2}}\right] .
$$

Using (35) and the condition $\bar{E}<0$ of the bound states, one can know that the condition for the charges that enable them to occupy the levels is $E<\hbar \omega / 4|m|$. Given a value of $|m|$, there are infinite states corresponding to the values of $n_{p}$ available. An alternative viewpoint for the condition can be given by the wave length. If we express the energy of the charge by $E=p^{2} / 2 M$, and express $p$ in terms of the wave length $\lambda$ of the matter wave, $p=h / \lambda$, the condition of a charge occupying a bound state in Equation (35) becomes

$$
\lambda>2|m| \lambda_{0},
$$

where $\lambda=\lambda / 2 \pi$ and $\lambda_{0}=\sqrt{M \omega / \hbar}$ is the characteristic length of the system of the simple harmonic oscillator. The radial wave function is now known as

$$
R(\bar{p}) \sim \bar{p}^{|\mu|} e^{-\beta \bar{p}} F\left(-n_{p}, 2|\mu|+1 ; 2 \beta \bar{p}\right) .
$$

This can also be expressed as

$$
R(\bar{p})=C \bar{p}|\mu| e^{-\beta \bar{p}} L_{n_{p}}^{2|\mu|}(2 \beta \bar{p})
$$

with $C$ being constant since $F\left(-n_{p}, 2|\mu|+1 ; 2 \beta \bar{p}\right) \sim L_{n_{p}}^{2|\mu|}(2 \beta \bar{p})$ the generalized Laguerre polynomials (p. 240, [12]). Employing the normalization condition,

$$
\int \Psi \Psi^{*} \bar{p} d \bar{p} d \bar{\varphi}=1
$$

the recurrence relation $L_{n}^{\alpha}=\left(L_{n}^{\alpha+1}-L_{n-1}^{\alpha+1}\right)$, and the orthogonal relation (p. 241, [12])

$$
\int_{0}^{\infty} y^{\alpha} e^{-y} L_{n}^{\alpha}(y) L_{m}^{\alpha}(y)=\frac{\Gamma(n+\alpha+1)}{n !} \delta_{n m},
$$

the radial wave function is found to be

$$
R(\bar{p})=2 \frac{\sqrt{|\mu| /(2 M \hbar|\omega|)}}{\left(n_{p}+|\mu|+1 / 2\right)} \sqrt{\frac{n_{p} !}{4 \pi\left(2 n_{p}+2|\mu|+1\right) \Gamma\left(n_{p}+2|\mu|+1\right)}} \times \xi|\mu| e^{-\xi / 2} L_{n_{p}}^{2|\mu|}(\xi) .
$$

Here the constant factor $\beta$ has been replaced by the explicit form $\beta=\sqrt{|\mu| /(2 M \hbar|\omega|)} /\left(n_{p}+|\mu|+1 / 2\right)$. Figures $1-3$, show the probability distributions of several bound states corresponding to the quantum number $\left(m, n_{p}\right)$. There are no corresponding states for a charge with $m=0$ in the transmutation system. This point is clear from the coupling constant (36). 


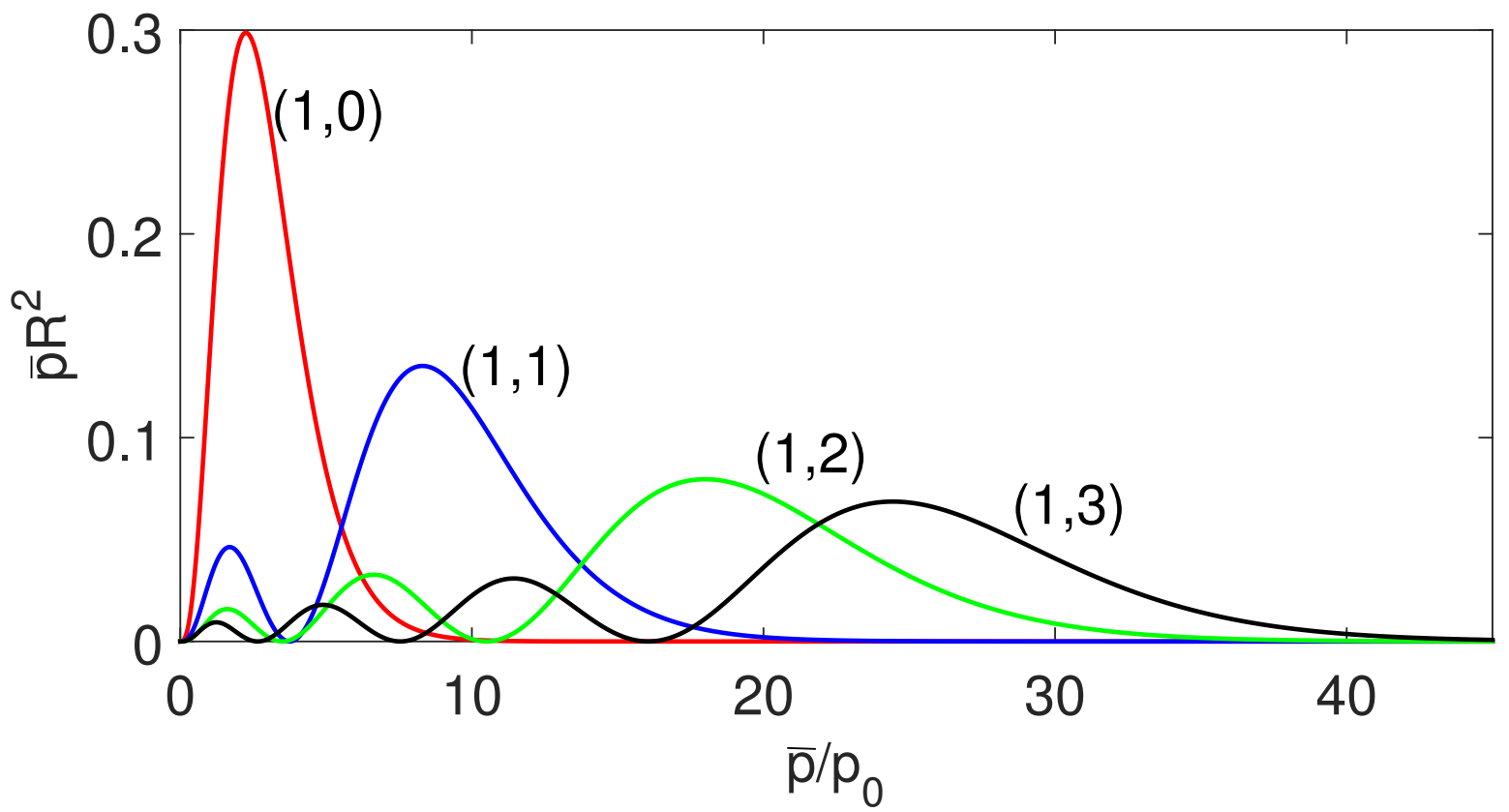

Figure 1. (Color online) The radial probability distribution $\bar{p} R^{2}(\bar{p})$ of the magnetic Hooke-Newton transmutation system in the momentum space. The distribution is classified by the quantum number $\left(|m|, n_{p}\right)$, where $m= \pm 1, \pm 2, \cdots$. Given several $m$ equivalent to giving the strength of the interaction, there is an infinite number of possible states corresponding to $n_{p}=0,1,2,3, \cdots$ available. The number of $n_{p}$ labels the number of the nodes in a distribution.

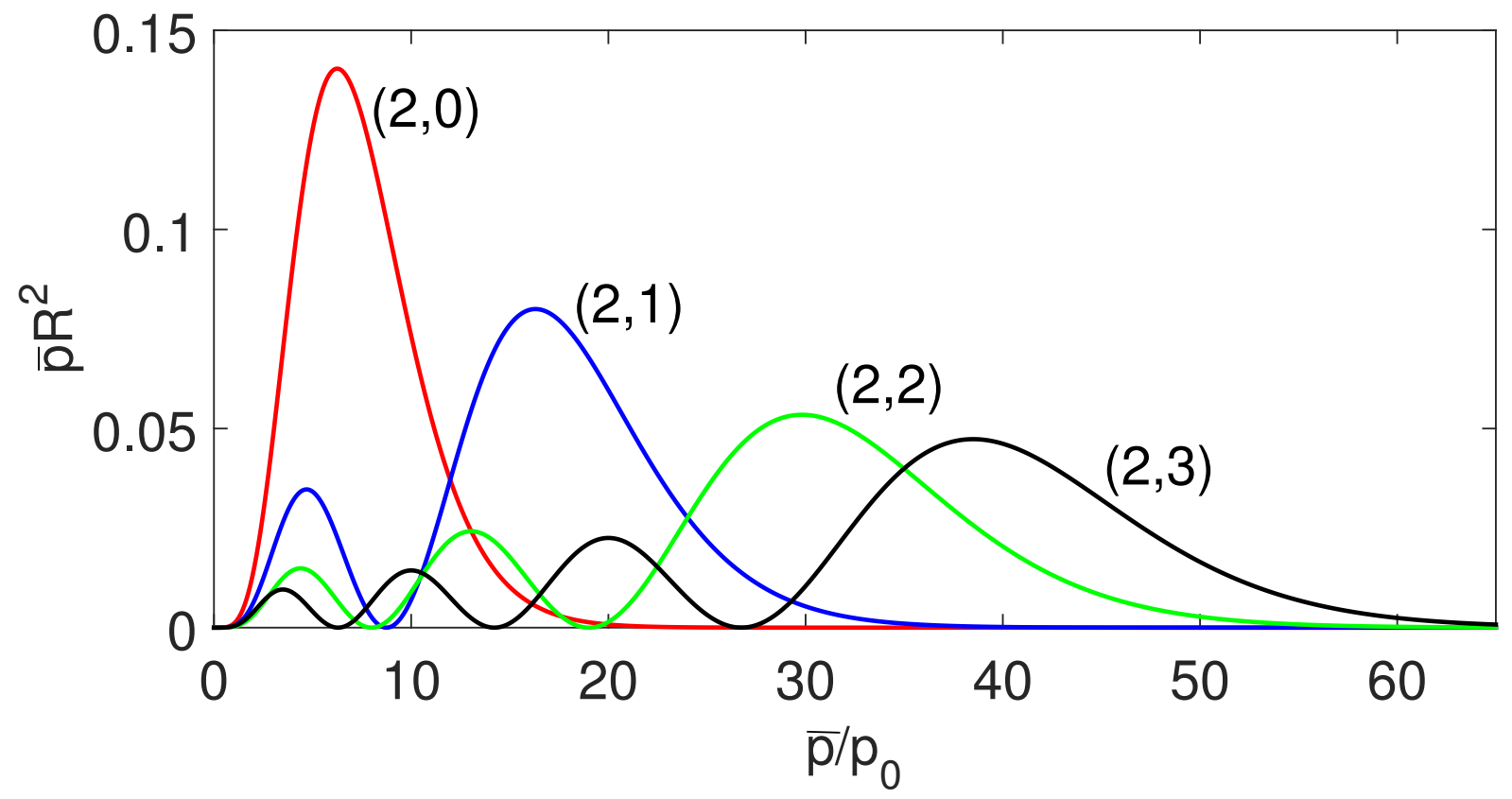

Figure 2. (Color online) The radial probability distribution of the pair $\left(2, n_{p}\right)$. 


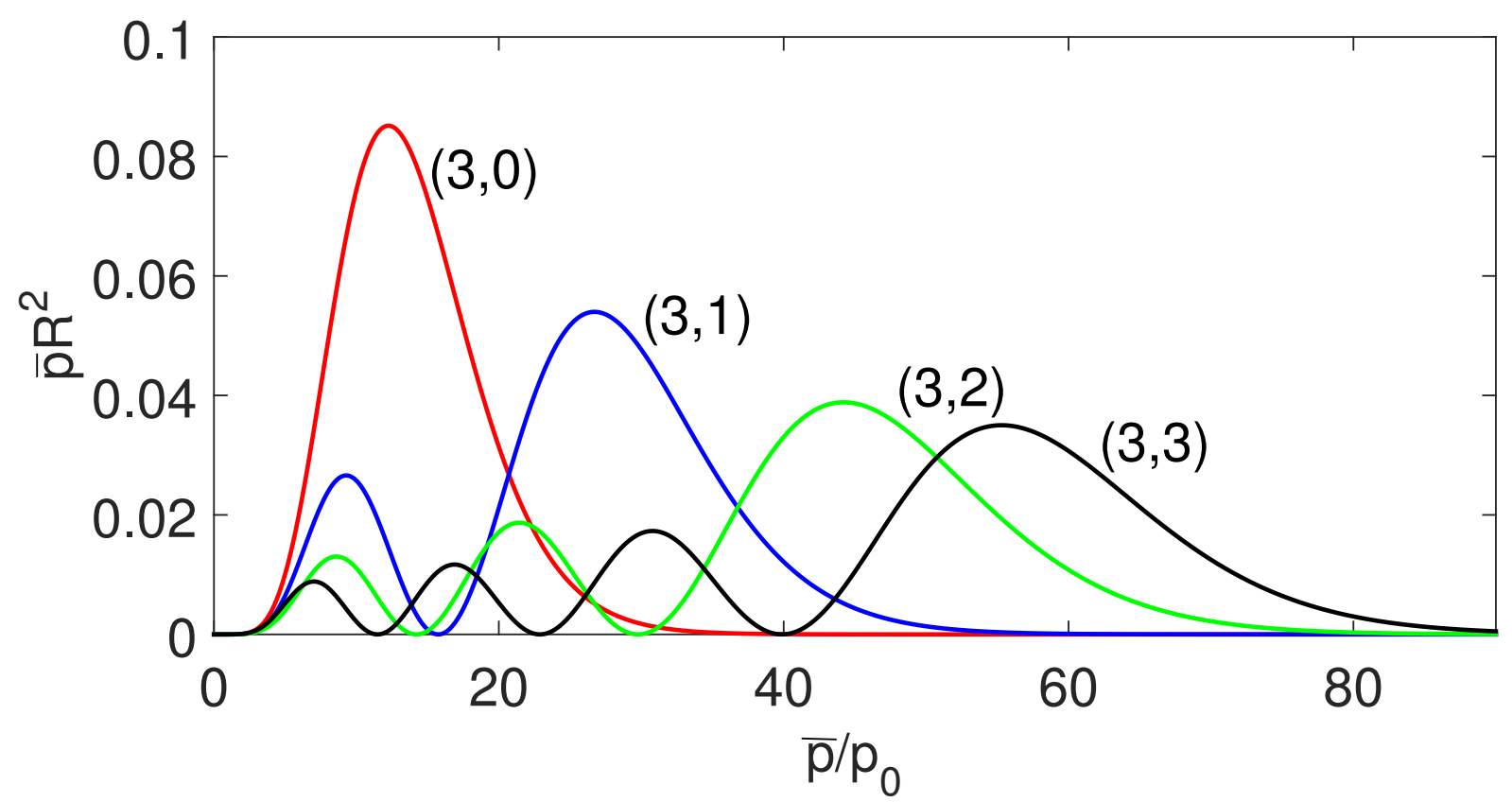

Figure 3. (Color online) The radial probability distribution of the pair $\left(3, n_{p}\right)$.

\subsection{Scattering State}

As shown in (38), a particle with a state of different signatures of the angular momentum and the charge in the transmutation system experiences the Coulomb-like repulsive force. It suffers scattering. The radial wave of the scattering state satisfies the same equation of (34), but with the constant $\alpha=p_{0} \mu /(M \hbar \omega)<0$ and non-quantized spectrum $\bar{E}>0$. So the parameters $\beta=\sqrt{-\bar{E}}=-i \sqrt{|\bar{E}|} \equiv-i \kappa, \xi=-2 i \kappa \bar{p}$, and we have the wave function for the scattering state

$$
R(\bar{p})=C \bar{p}^{|\mu|} \cdot e^{i \kappa \bar{p}} \cdot F(|\mu|+1 / 2+i \gamma, 2|\mu|+1 ;-2 i \kappa \bar{p}),
$$

where $\gamma=|\alpha| /(2 \sqrt{|\bar{E}|})$. It is of interest to know the asymptotic representation of a scattering state as $\bar{p} \rightarrow \infty$. Using the asymptotic formula of the confluent hypergeometric function (p. 611, [19])

$$
F(a, b, \xi) \rightarrow \frac{\Gamma(b)}{\Gamma(a)}|\xi|^{(a-b)} e^{-i \pi(a-b) / 2} e^{\xi}+\frac{\Gamma(b)}{\Gamma(b-a)}|\xi|^{-a} e^{-i a \pi / 2}
$$

when $\xi=|\xi| e^{-i \pi / 2}$ and $|\xi| \rightarrow \infty$, and defining the phase shift of the scattering state by

$$
\Gamma(|\mu|+1 / 2+i \gamma)=|\Gamma(|\mu|+1 / 2+i \gamma)| e^{i \delta|\mu|}
$$

such that

$$
\frac{\Gamma(|\mu|+1 / 2+i \gamma)}{\Gamma(|\mu|+1 / 2-i \gamma)}=e^{2 i \delta|\mu|},
$$

we have the representation at the far region

$$
R(\bar{p}) \rightarrow \frac{\Gamma(2|\mu|+1)}{\Gamma(|\mu|+1 / 2+i \gamma)} \frac{e^{\pi \gamma / 2+i \delta|\mu|}}{(2 \kappa)^{|\mu|}} \frac{1}{\sqrt{2 \kappa \bar{p}}} \times 2 \cos \left[-\gamma \ln (2 \kappa \bar{p})-\frac{\pi|\mu|}{2}-\frac{\pi}{4}+\delta_{|\mu|}+\kappa \bar{p}\right] .
$$

The energy spectrum of the bound state can also be obtained from the analytic property of the representation. The poles of the scattering amplitude are at

$$
|\mu|+\frac{1}{2}+i \gamma=0,-1,-2, \cdots,
$$


which shows

$$
\gamma=\text { in, with } n=n_{p}+|\mu|+\frac{1}{2},
$$

and $n_{p}=0,1,2, \cdots$. Since

$$
|\bar{E}|=\left(\frac{|\alpha|}{2 \gamma}\right)^{2}=-\frac{(|\alpha| / 2)^{2}}{\left(n_{p}+|\mu|+1 / 2\right)^{2}},
$$

substitution (35) in the equality gives the quantization levels in (45) again.

\section{Conclusions}

This paper investigates the magnetic Hooke-Newton transmutation in the momentum space. On the one hand, it shows the effectiveness of the transformation design method in a representation space other than the coordinate space that may enlarge the application of the representation theory. On the other hand, it clarifies the difference between the transmuted Coulomb systems by directly performing the quadratic conformal mapping with respect to the equation of the Landau level problem and that presented in the recent works due to the transformation design which exhibits a novel quantum spectrum. The latter includes the action of an effective potential defined through the conformal structure of the mapping, while the former does not. Three remarks are worth making as follows:

(a) The transmuted Coulomb system due to the transformation design in the momentum space intrinsically differs from the common Coulomb system since the dynamics of the usual Coulomb system in the momentum space exhibit its behavior as the free particle on the unit sphere (e.g., [20]). Our presentation shows that the transmuted Coulomb system is caused by the action of the effective potential which is determined by the geometric structure of considered representation spaces. It has the same form either in the momentum or in the coordinate space.

(b) The potential effect coming from the geometric structure fractionalizes and turns down the ground state energy $\hbar \omega$ of the charged particle in the uniform magnetic field. The quantum number $m$ determines the coupling strength of interaction as shown in (36). A series of energy levels are physically allowed after being given $m$. The energy of the ground state of the given $m$ reads

$$
E_{m 0}=\frac{\hbar \omega}{4|m|}\left[1-\frac{m^{2}}{(|m|+1 / 2)^{2}}\right],
$$

where the suffix labels the quantum number $\left(m, n_{p}\right)$, which fractionalizes $\hbar \omega$. The ground state energy never comes to zero in any value of finite $m$, and the energy levels of the particle grow with the increase of the radial quantum number $n_{p}$. Experimentally, a better way for the observation of the effect of the levels in Equation (45) is through offering the potential field

$$
U=\left(1-\frac{4 \rho^{2}}{\rho_{0}^{2}}\right) E
$$

to the Landau level system in the coordinate space, where $\rho_{0}=\sqrt{4 \hbar /\left(|m| M \omega_{c}\right)}$, as given above (44), and the energy $E$ should be chosen as the highest critical energy $E=\hbar \omega /(4|m|)$ of the bound states to have enough attractive force in the region of $2 \rho>\rho_{0}$ to trap the charges with lower energy. The system under the quadratic conformal mapping has the same spectrum as (45). For $m=1$, the spectrum has the lowest energy level $E_{10}=5 \hbar \omega / 36$, and the level $E_{11}=21 \hbar \omega / 100$ of the first excited state. Therefore, the energy of an emitted photon generated by the transition from $E_{11}$ to the ground state is

$$
E_{11}-E_{10}=\frac{16}{225} \hbar \omega .
$$


For an electron with charge $q=-e$ and the magnetic field $B=1$ Tesla, $\hbar \omega \approx 0.58 \times 10^{-4} \mathrm{eV}$. With the definite value of $\hbar \omega$, the constant $\rho_{0}$ in the potential $U$ can be evaluated. We find $\rho_{0} \approx 5.1 \times 10^{-2} / \sqrt{|m|} \mu \mathrm{m}$ such that the potential field can be set up in the laboratory. The energy difference is now given by $E_{11}-E_{10}=4.12 \times 10^{-6} \mathrm{eV}$ which corresponds to the wave length $\lambda=3.01 \times 10^{9} \AA$. It is about the range of microwaves, and should not be difficult to observe using the technique of the spectroscopy.

(c) The phase transition and symmetry breaking are important topics (e.g., [21-24]). Two points for the ground state of a $2 \mathrm{D}$ system generated by the processes are stated as follows: (i) The behavior of the ground state would not be altered by the quadratic conformal mapping. The reason is as follows: If the interaction in the system can be described by a potential and has ground state, it stands for the potential of the system has minimum to support the ground state. Since the potential at its minimum can always be expanded and approximated by the harmonic trap potential, the question comes a similar appearance to the section II of the paper where the ground state of the potential of the harmonic trap under the quadratic conformal mapping does not alter by the mapping as shown in Equation (31). (ii) If the system with ground state is added by the effective potential presented in (61), the level of the ground state would be lowered under the quadratic conformal mapping as shown in Equation (45) in Section 3. The cause is due to the fact that the effective potential $U=\left(1-4 \rho^{2} / \rho_{0}^{2}\right) E$ offers an additional attractive (repulsive) force when $4 \rho^{2}>\rho_{0}^{2}\left(4 \rho^{2}<\rho_{0}^{2}\right)$. The attractive region would lower the ground state in the target space of conformal mapping. On the other hand, the repulsive region helps to establish the possible scattering state in the spectrum of the transmutation system such as the result shown in Section 3. For a general conformal mapping, the influence on the ground state needs to be further investigated.

Funding: This research received no external funding.

Institutional Review Board Statement: Not applicable.

Informed Consent Statement: Not applicable.

Data Availability Statement: Not applicable.

Acknowledgments: The author thanks C. R. Harrington for her critical reading of the manuscript. My work has been supported by the Ministry of Science and Technology of Taiwan under Contract No. MOST 108-2112-M-110-008-MY2.

Conflicts of Interest: The author declares no conflict of interest.

\section{Appendix A. The Conformal Structure of the Form-Invariant Line Element and the Effective Potential}

Here we prove that the quadratic conformal mapping turns the Schrödinger equation of a particle interacting with the effective potential defined by the conformal structure of the 2D p-space into the form-invariant Schrödinger equation of the free particle on the 2D plane. Consider the form-invariant line element in the 2D p-space

$$
d \bar{s}^{2}=d \bar{p}^{2}+\bar{p}^{2} d \bar{\varphi}^{2}
$$

It is assumed that $\bar{p}=\bar{p}(p)$ and $\bar{\varphi}=\bar{\varphi}(\varphi)$. The line element has the equivalent expression

$$
d \bar{s}^{2}=\left(\frac{d \bar{p}}{d p}\right)^{2} d p^{2}+\left(\frac{\bar{p} d \bar{\varphi}}{p d \varphi}\right)^{2} p^{2} d \varphi^{2} .
$$

The condition of the line element (A1) generated by a conformal mapping is

$$
\frac{d \bar{p}}{d p}=\frac{\bar{p} d \bar{\varphi}}{p d \varphi} .
$$


This is equivalent to the statement

$$
\frac{p d \bar{p}}{\bar{p} d p}=\frac{d \bar{\varphi}}{d \varphi}=v
$$

with $v$ being a constant. So we have the mapping

$$
\bar{p}=\frac{p^{v}}{p_{0}^{v-1}}, \text { and } \bar{\varphi}=\nu \varphi,
$$

where $p_{0}$ is a constant with the units of momentum used to balance the units. Accordingly, $d \bar{s}$ has the conformal representation

$$
d \bar{s}^{2}=\left(\bar{p}^{\prime}\right)^{2}\left(d p^{2}+p^{2} d \varphi^{2}\right)=\left(\bar{p}^{\prime}\right)^{2} d s^{2} .
$$

Through the conformal factor, define the effective potential

$$
U=[1-\text { conformal factor }] E=\left[1-\left(\bar{p}^{\prime}\right)^{2}\right] E .
$$

The Schrödinger equation in the polar coordinates with the action of the potential is given by

$$
\frac{-\hbar^{2}}{2 A}\left[\frac{\partial^{2}}{\partial p^{2}}+\frac{1}{p} \frac{\partial}{\partial p}+\frac{1}{p^{2}} \frac{\partial^{2}}{\partial \varphi^{2}}\right] \Psi=(E-U) \Psi .
$$

The relations between the derivatives of the variables $(p, \varphi)$, and $(\bar{p}, \bar{\varphi})$ read

$$
\frac{\partial}{\partial p}=\bar{p}^{\prime} \frac{\partial}{\partial \bar{p}^{\prime}}, \frac{\partial^{2}}{\partial p^{2}}=\bar{p}^{\prime \prime} \frac{\partial}{\partial \bar{p}}+\left(\bar{p}^{\prime}\right)^{2} \frac{\partial}{\partial \bar{p}^{\prime}} \text {, and } \frac{\partial^{2}}{\partial \varphi^{2}}=v^{2} \frac{\partial^{2}}{\partial \bar{\varphi}^{2}} .
$$

With $\bar{p}^{\prime}=v \bar{p} / p$, and $\bar{p}^{\prime \prime}=v(v-1) \bar{p} / p^{2}$, the replacement of (A9) in (A8) results in the form-invariant Schrödinger equation of the free particle

$$
\frac{-\hbar^{2}}{2 A}\left[\frac{\partial^{2}}{\partial \bar{p}^{2}}+\frac{1}{\bar{p}} \frac{\partial}{\partial \bar{p}}+\frac{1}{\bar{p}^{2}} \frac{\partial^{2}}{\partial \bar{\varphi}^{2}}\right] \Psi=E \Psi .
$$

The statement above is confirmed.

Using the geometric structure of the conformal mapping to define the effective potential can be generalized to a general 2D surface. The conformal mapping of the surface has the efficiency of turning the Schrödinger equation of a particle under the action of the potential into the form-invariant equation of the free particle on the surface. The solution of the particle with the interaction on the surface can thus be solved by the form-invariant solution of the free particle. The verification of the statement is as follows. Consider a 2D surface in the p-space described by the line element

$$
d s^{2}=g_{11}\left(p_{2}\right)\left(d p_{1}\right)^{2}+g_{22}\left(p_{1}\right)\left(d p_{2}\right)^{2}
$$

Define a form-invariant element by

$$
d \bar{s}^{2}=\bar{g}_{11}\left(\bar{p}_{2}\right)\left(d \bar{p}_{1}\right)^{2}+\bar{g}_{22}\left(\bar{p}_{1}\right)\left(d \bar{p}_{2}\right)^{2}
$$

with $\bar{p}_{i}=\bar{p}_{i}\left(p_{i}\right)$ only. It can also be expressed as

$$
d \bar{s}^{2}=\frac{\bar{g}_{11}\left(\bar{p}_{2}\right)\left(\bar{p}_{1}^{\prime}\right)^{2}}{g_{11}\left(p_{2}\right)} g_{11}\left(p_{2}\right)\left(d p_{1}\right)^{2}+\frac{\bar{g}_{22}\left(\bar{p}_{1}\right)\left(\bar{p}_{2}^{\prime}\right)^{2}}{g_{22}\left(p_{1}\right)} g_{22}\left(p_{1}\right)\left(d p_{2}\right)^{2} .
$$

Here $\bar{p}_{i}^{\prime}=d \bar{p}_{i} / d p_{i}$. The condition of the line element (A12) generated by conformal mapping is 


$$
\frac{\bar{g}_{11}\left(\bar{p}_{2}\right)\left(\bar{p}_{1}^{\prime}\right)^{2}}{g_{11}\left(p_{2}\right)}=\frac{\bar{g}_{22}\left(\bar{p}_{1}\right)\left(\bar{p}_{2}^{\prime}\right)^{2}}{g_{22}\left(p_{1}\right)} .
$$

So

$$
d \bar{s}^{2}=\left[\frac{\bar{g}_{i i}\left(\bar{p}_{j}\right)\left(\bar{p}_{i}^{\prime}\right)^{2}}{g_{i i}\left(p_{j}\right)}\right] d s^{2},
$$

where $i, j=1,2$ and $i \neq j$. Equation (A14) is equivalent to

$$
\frac{\bar{g}_{11}\left(\bar{p}_{2}\right)}{g_{11}\left(p_{2}\right)\left(\bar{p}_{2}^{\prime}\right)^{2}}=\frac{\bar{g}_{22}\left(\bar{p}_{1}\right)}{g_{22}\left(p_{1}\right)\left(\bar{p}_{1}^{\prime}\right)^{2}}=C,
$$

with $C$ being an arbitrary constant which shows

$$
\bar{g}_{i i}\left(\bar{p}_{j}\right)=C g_{i i}\left(p_{j}\right)\left(\bar{p}_{j}^{\prime}\right)^{2},
$$

and the determinants of two kinds of metric are associated with the equality

$$
\sqrt{\bar{g}}=\sqrt{\operatorname{det}\left|\bar{g}_{i i}\right|}=C \bar{p}_{1}^{\prime} \bar{p}_{2}^{\prime} \sqrt{g} .
$$

The state of a particle moving on an arbitrary 2D surface with orthogonal frame (A11) while experiencing the action of the potential $U$ satisfies the equation

$$
-\frac{\hbar^{2}}{2 A} \frac{1}{\sqrt{g}} \sum_{i} \frac{\partial}{\partial p_{i}}\left(\sqrt{g} g^{i i} \frac{\partial}{\partial p_{i}} \Psi\right)=(E-U) \Psi,
$$

where

$$
U=\left[1-\frac{\bar{g}_{i i}\left(\bar{p}_{j}\right)\left(\bar{p}_{i}^{\prime}\right)^{2}}{g_{i i}\left(p_{j}\right)}\right] E .
$$

Inserting the identities $\sqrt{\bar{g} / \bar{g}}=1$ and $\sqrt{\bar{g}} \bar{g}^{i i} / \sqrt{\bar{g}} \bar{g}^{i i}=1$ into the equation makes it become

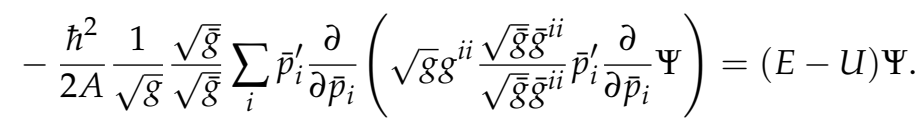

Using (A17) and (A18), it can reduce the factor in brackets,

$$
\frac{\sqrt{g} g^{i i}}{\sqrt{\bar{g}} \bar{g} i i} \bar{p}_{i}^{\prime}=\frac{\sqrt{g} \bar{g}_{i i}}{\sqrt{\bar{g}} g_{i i}} \bar{p}_{i}^{\prime}=\bar{p}_{j}^{\prime}, i \neq j .
$$

The summation terms become

$$
\sum_{i} \bar{p}_{i}^{\prime} \bar{p}_{j}^{\prime} \frac{\partial}{\partial \bar{p}_{i}}(\cdots)=\bar{p}_{i}^{\prime} \bar{p}_{j}^{\prime} \sum_{i} \frac{\partial}{\partial \bar{p}_{i}}\left(\sqrt{\bar{g}} \bar{g}^{i i} \frac{\partial}{\partial \bar{p}_{i}} \Psi\right) .
$$

Equation (A21) reduces to

$$
-\frac{\hbar^{2}}{2 A}\left(\frac{\sqrt{\bar{g}}}{\sqrt{g}} \bar{p}_{i}^{\prime} \bar{p}_{j}^{\prime}\right) \frac{1}{\sqrt{\bar{g}}} \sum_{i} \frac{\partial}{\partial \bar{p}_{i}}\left(\sqrt{\bar{g}} \bar{g}^{i i} \frac{\partial}{\partial \bar{p}_{i}} \Psi\right)=\frac{\bar{g}_{i i}\left(\bar{p}_{j}\right)\left(\bar{p}_{i}^{\prime}\right)^{2}}{g_{i i}\left(p_{j}\right)} E \Psi .
$$

Using (A17) and (A18) again, it is easy to prove

$$
\left(\frac{\sqrt{\bar{g}}}{\sqrt{g}} \bar{p}_{i}^{\prime} \bar{p}_{j}^{\prime}\right)=\frac{\bar{g}_{i i}\left(\bar{p}_{j}\right)\left(\bar{p}_{i}^{\prime}\right)^{2}}{g_{i i}\left(p_{j}\right)},
$$


and the equation becomes the form-invariant Schrödinger equation

$$
-\frac{\hbar^{2}}{2 A} \frac{1}{\sqrt{\bar{g}}} \sum_{i} \frac{\partial}{\partial \bar{p}_{i}}\left(\sqrt{\bar{g}} \bar{g}^{i i} \frac{\partial}{\partial \bar{p}_{i}} \Psi\right)=E \Psi .
$$

It is obvious that the discussion and conclusion are true in any representation space. As an application, let us construct the form-invariant equation on the hyperbolic surface $\left(\mathrm{H}^{2}\right)$ and discuss its quantum mechanics. The Riemannian metric on the surface can be expressed as

$$
d s^{2}=d u^{2}+u_{0}^{2} \sinh ^{2}\left(\frac{u}{u_{0}}\right) d \theta^{2},
$$

where $0 \leq u<\infty, 0 \leq \theta \leq 2 \pi$, and $u_{0}>0$ is a constant. The negative constant curvature of the surface is given by $K=-1 / u_{0}^{2}$. Consider the form-invariant metric

$$
d \bar{s}^{2}=d \bar{u}^{2}+u_{0}^{2} \sinh ^{2}\left(\frac{\bar{u}}{u_{0}}\right) d \bar{\theta}^{2},
$$

with $\bar{u}=\bar{u}(u)$ and $\bar{\theta}=\bar{\theta}(\theta)$. The equalities in (A16) imply

$$
\frac{d \bar{\theta}}{d \theta}=\frac{d \bar{u}}{d u} \frac{\sinh \left(u / u_{0}\right)}{\sinh \left(\bar{u} / u_{0}\right)}=a \text {, with } a=\text { constant. }
$$

Here we just take $C=1 / a^{2}$ in (A16). The explicit representation of the form-invariant coordinates $(\bar{u}, \bar{\theta})$ are found by integrations. They are

$$
\bar{\theta}=a \theta, \text { and } \tanh \left(\frac{\bar{y}}{2}\right)=\left(\tanh \frac{y}{2}\right)^{a},
$$

where the dimensionless variables $\bar{y}=\bar{u} / u_{0}$ and $y=u / u_{0}$ were introduced for short. Obviously, the variable $y$ has the same range as $u$. With the help of the half angle formula $\tanh ^{2} x / 2=(\cosh x-1) /(\cosh x+1)$, the transformation relation between $\bar{y}$ and $y$ has the expression

$$
\cosh \bar{y}=\frac{(\cosh y+1)^{a}+(\cosh y-1)^{a}}{(\cosh y+1)^{a}-(\cosh y-1)^{a}},
$$

or

$$
\sinh \bar{y}=\frac{2(\sinh y)^{a}}{(\cosh y+1)^{a}-(\cosh y-1)^{a}} .
$$

Both functions are anti-symmetric with respect to the index $-a$, i.e.,

$$
\left.\cosh \bar{y}\right|_{-a}=-\left.\cosh \bar{y}\right|_{a} \text {, and }\left.\sinh \bar{y}\right|_{-a}=-\left.\sinh \bar{y}\right|_{a} .
$$

Figures A1 and A2 show some graphs of the hyperbolic functions where the range of the transverse $y$-axis was extended to the negative value for exhibiting their symmetric properties. It is easy to verify that the familiar relationship $\cosh ^{2} \bar{y}-\sinh ^{2} \bar{y}=1$ of the hyperbolic functions holds. Moreover, the derivative relations 


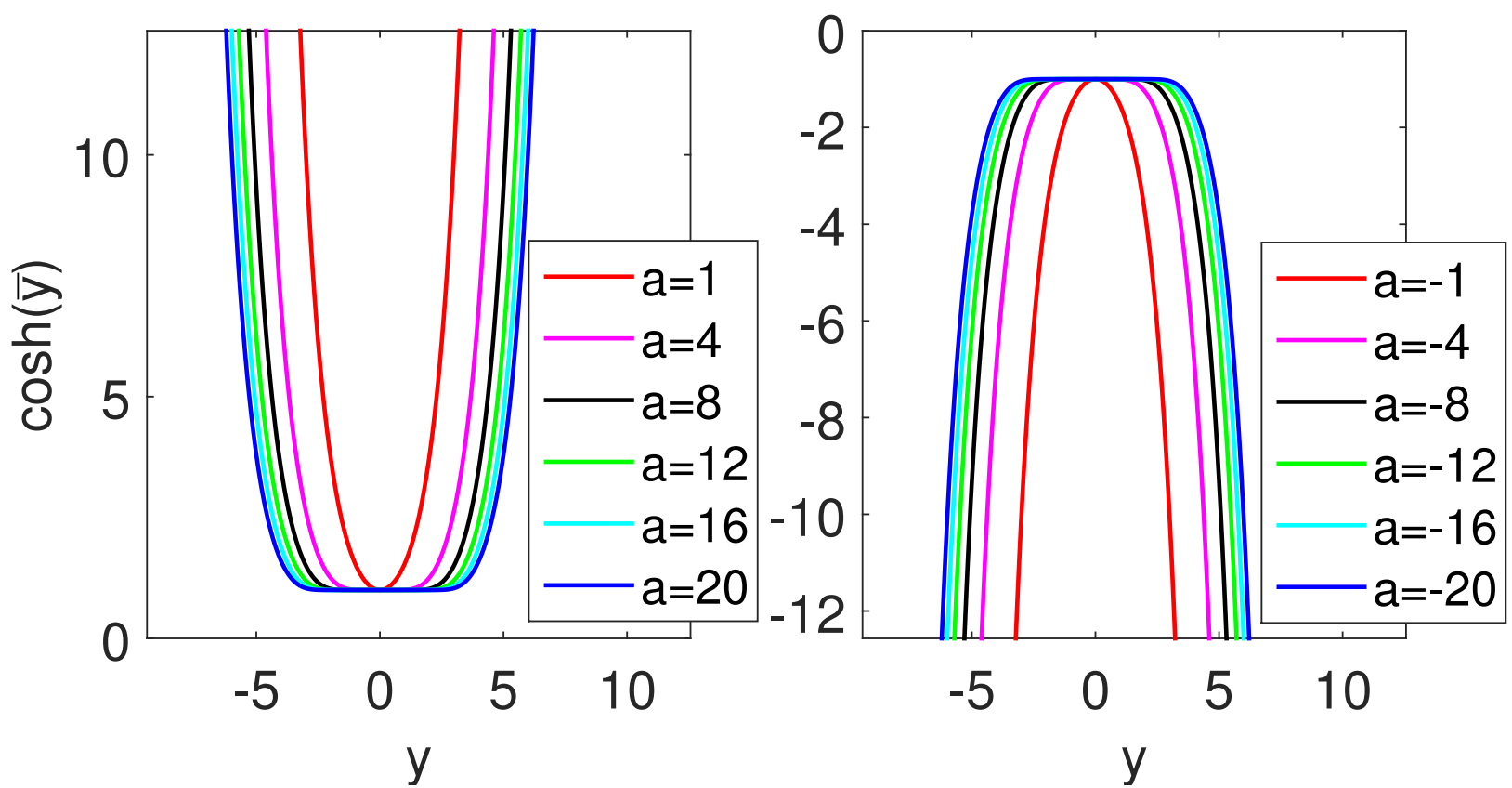

Figure A1. (Color online) The graphs of the hyperbolic function $\cosh (\bar{y})$ for some values of the conformal index $a$ in (A31). Here the range of the transverse $y$-axis is extended to the negative values for the exhibition of the symmetric property of the function.

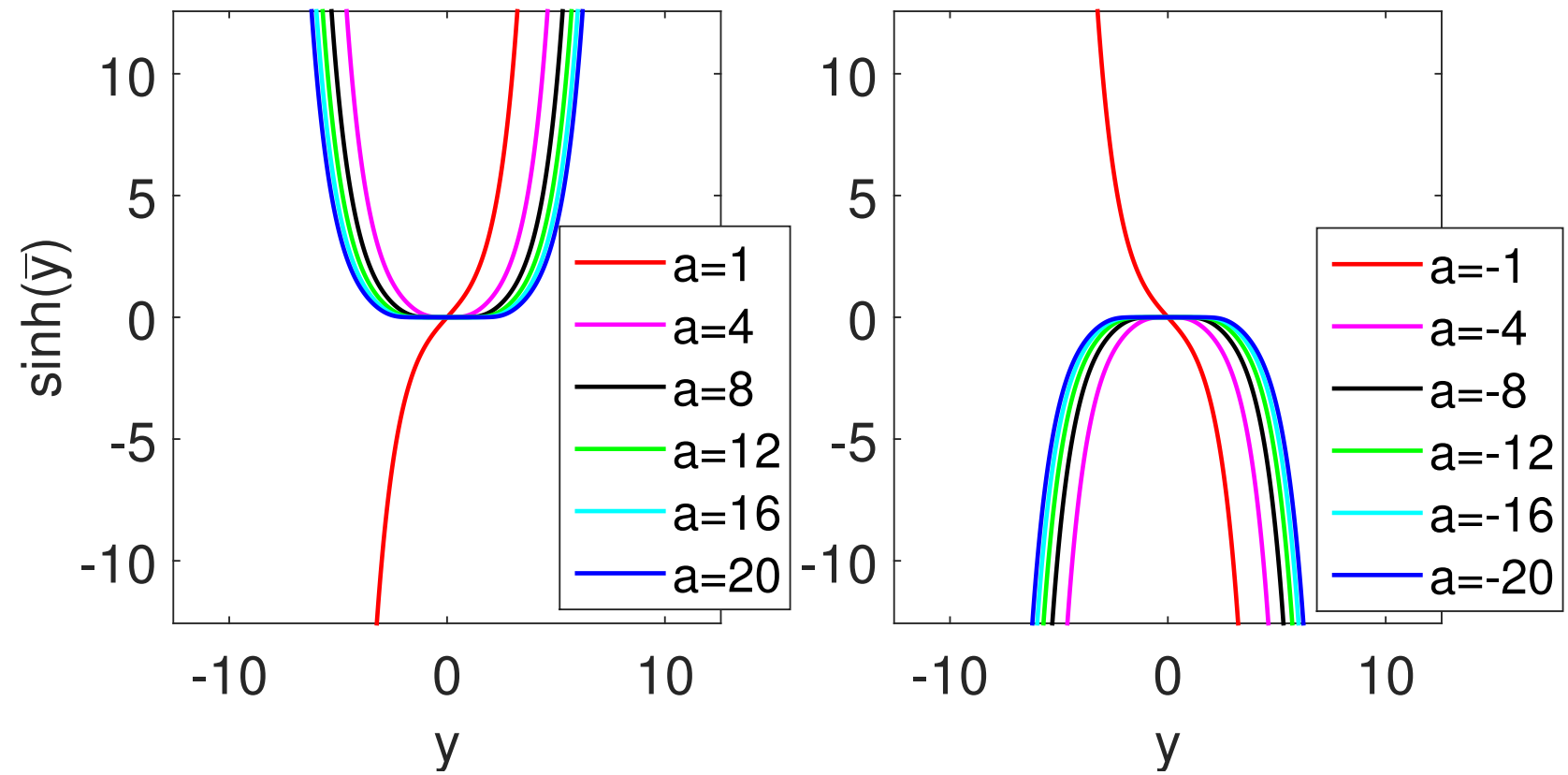

Figure A2. (Color online) The graphs of the hyperbolic function $\sinh (\bar{y})$ for some values of $a$ in (A32). Both functions, $\cosh (\bar{y})$ and $\sinh (\bar{y})$, are anti-symmetric to the negative values of $a$.

$$
\frac{d}{d \bar{y}} \sinh \bar{y}=\frac{d y}{d \bar{y}} \frac{d}{d y} \sinh \bar{y}=\cosh \bar{y}
$$

and

$$
\frac{d}{d \bar{y}} \cosh \bar{y}=\frac{d y}{d \bar{y}} \frac{d}{d y} \cosh \bar{y}=\sinh \bar{y}
$$


are also true, where

$$
\frac{d \bar{y}}{d y}=\frac{2 a(\sinh y)^{a-1}}{(\cosh y+1)^{a}-(\cosh y-1)^{a}}=\frac{a \sinh \bar{y}}{\sinh y} .
$$

The transformations (A30) and (A32) are conformal mapping. This is confirmed by the conformal condition

$$
\frac{d \bar{\theta}}{d \theta}=\frac{d \bar{u}}{d u} \frac{\sinh \left(u / u_{0}\right)}{\sinh \left(\bar{u} / u_{0}\right)}=\frac{d \bar{y}}{d y} \frac{\sinh (y)}{\sinh (\bar{y})}
$$

The form-invariant metric (A28) is associated with $d s^{2}$ by a conformal factor $\left(\bar{u}^{\prime}\right)^{2}$ as

$$
d \bar{s}^{2}=\left(\bar{u}^{\prime}\right)^{2}\left[d u^{2}+u_{0}^{2} \sinh ^{2}\left(\frac{u}{u_{0}}\right) d \theta^{2}\right]=\left(\bar{u}^{\prime}\right)^{2} d s^{2},
$$

where $\bar{u}^{\prime}=d \bar{u} / d u=d \bar{y} / d y=\bar{y}^{\prime}$. It can be proved that the conformal space has the same Gauss curvature $K=-1 / u_{0}^{2}$ as $H^{2}$. Since the metric of $H^{2}$ is $\left(g_{i i}\right)=\operatorname{diag}\left(1, u_{0}^{2} \sinh ^{2}\left(u / u_{0}\right)\right)$, the form-invariant metrics of the conformal surface of $\mathrm{H}^{2}$ are

$$
\begin{gathered}
\bar{g}_{11}=C\left(\bar{\theta}^{\prime}\right)^{2} g_{11}=1, \\
\bar{g}_{22}=C\left(\bar{u}^{\prime}\right)^{2} g_{22}=u_{0}^{2} \sinh ^{2} \bar{y},
\end{gathered}
$$

where $\left(\bar{u}^{\prime}\right)^{2}=\left(\bar{y}^{\prime}\right)^{2}=(a \sinh \bar{y} / \sinh y)^{2}$ and $C=1 / a^{2}$ were used. Accordingly, we get the form-invariant Schrödinger equation on $\mathrm{H}^{2}$

$$
-\frac{\hbar^{2}}{2 M u_{0}^{2}}\left[\frac{1}{\sinh \bar{y}} \frac{\partial}{\partial \bar{y}}\left(\sinh \bar{y} \frac{\partial}{\partial \bar{y}}\right)+\frac{1}{\sinh ^{2} \bar{y}} \frac{\partial^{2}}{\partial \bar{\theta}^{2}}\right] \Psi=E \Psi
$$

from (A26). It describes the evolution of a quantum particle moving on $\mathrm{H}^{2}$ while it experiences the action of the potential field

$$
U=\left[1-\left(\bar{u}^{\prime}\right)^{2}\right] E .
$$

The wave function permits the factorization $\Psi=R(\bar{y}) e^{i \mu \bar{\theta}}$ such that the radial wave $R(\bar{y})$ satisfies the equation

$$
\left[\frac{1}{\sinh \bar{y}} \frac{d}{d \bar{y}}\left(\sinh \bar{y} \frac{d}{d \bar{y}}\right)+\left(k^{2}-\frac{\mu^{2}}{\sinh ^{2} \bar{y}}\right)\right] R=0 .
$$

Here $k^{2}=2 M E u_{0}^{2} / \hbar^{2}$. If we perform a transformation of $R$ by $R=F(\bar{y}) / \sqrt{\sinh \bar{y}}$, the equation reduces to

$$
\frac{d^{2}}{d \bar{y}^{2}} F+\left[\lambda^{2}-\left(\frac{\mu^{2}-1 / 4}{\sinh ^{2} \bar{y}}\right)\right] F=0
$$

where $\lambda^{2}=\left(k^{2}-1 / 4\right)$. The value of $\mu$ is determined by the single-valuedness condition of the wave function which means $e^{i \mu \bar{\theta}}=e^{i \mu(\bar{\theta}+2 \pi)}$, implying that $\mu$ is an arbitrary integer

$$
\mu=n_{1}, n_{1}=0, \pm 1, \pm 2, \cdots
$$

Notice the existence of the bound states only if $\mu^{2}<1 / 4$. Then, the potential would offer an attractive force on the particle to form the bound states. For the $\mathrm{H}^{2}$ surface, it was 
supposed to only have the scattering states (e.g., [25]). To solve the equation, introduce the new variable $\bar{x}=-\sinh ^{2} \bar{y}$ turning (A44) into

$$
\bar{x}(1-\bar{x}) \frac{d^{2} F}{d \bar{x}^{2}}+\left(\frac{1}{2}-\bar{x}\right) \frac{d F}{d \bar{x}}-\left[\left(\frac{\lambda}{2}\right)^{2}+\frac{\eta(\eta-1)}{4 \bar{x}}\right] F=0,
$$

where $\eta=(|\mu|+1 / 2)$. If we perform the transformation $F(\bar{x})=\bar{x}^{\eta / 2} G(\bar{x})$, we find that $G(\bar{x})$ satisfies the equation

$$
\bar{x}(1-\bar{x}) \frac{d^{2} G}{d \bar{x}^{2}}+\left[\left(\eta+\frac{1}{2}\right)-(\eta+1) \bar{x}\right] \frac{d G}{d \bar{x}}-\frac{1}{4}\left(\eta^{2}+\lambda^{2}\right) G=0 .
$$

Put

$$
b_{1}=\frac{1}{2}(\eta+i \lambda), b_{2}=\frac{1}{2}(\eta-i \lambda), \text { and } c_{1}=\left(\eta+\frac{1}{2}\right) .
$$

The equation becomes the form of the hypergeometric equation (e.g., p. 272, [26])

$$
\bar{x}(1-\bar{x}) \frac{d^{2} G}{d \bar{x}^{2}}+\left[c_{1}-\left(b_{1}+b_{2}+1\right) \bar{x}\right] \frac{d G}{d \bar{x}}-b_{1} b_{2} G=0 .
$$

One solution of the equation is the hypergeometric function ${ }_{2} F_{1}\left(b_{1}, b_{2} ; c_{1} ; \bar{x}\right)$. Therefore, the solution of (A44) is

$$
F=\bar{x}^{\eta / 2} G(\bar{x}) \sim(-\sinh \bar{y})^{\eta}{ }_{2} F_{1}\left(\frac{(\eta+i \lambda)}{2}, \frac{(\eta-i \lambda)}{2} ;\left(\eta+\frac{1}{2}\right) ;-\sinh ^{2} \bar{y}\right) .
$$

A convenient way to obtain the energy levels is through the positions of the pole of the scattering wave. For this, using the asymptotic formula of the hypergeometric function at $z \rightarrow \infty$ (e.g., p. 273, [26])

$$
{ }_{2} F_{1}\left(b_{1}, b_{2} ; c_{1} ; z\right) \longrightarrow \frac{\Gamma\left(c_{1}\right) \Gamma\left(b_{2}-b_{1}\right)}{\Gamma\left(b_{2}\right) \Gamma\left(c_{1}-b_{1}\right)}(-z)^{-b_{1}}+\frac{\Gamma\left(c_{1}\right) \Gamma\left(b_{1}-b_{2}\right)}{\Gamma\left(b_{1}\right) \Gamma\left(c_{1}-b_{2}\right)}(-z)^{-b_{2}},
$$

and noting that $-\sinh ^{2} \bar{y} \rightarrow-e^{2 \bar{y}} / 4$, the wave $F$ has the asymptotic representation

$$
\begin{gathered}
F \longrightarrow(-1)^{\eta / 2} \Gamma\left(\eta+\frac{1}{2}\right)\left[\frac{e^{i \lambda \ln 2} \Gamma(-i \lambda)}{\Gamma((\eta-i \lambda) / 2) \Gamma((\eta+1) / 2-i \lambda / 2)} e^{-i \lambda \bar{y}}\right. \\
\left.+\frac{e^{-i \lambda \ln 2} \Gamma(i \lambda)}{\Gamma((\eta+i \lambda) / 2) \Gamma((\eta+1) / 2+i \lambda / 2)} e^{i \lambda \bar{y}}\right]
\end{gathered}
$$

When $k^{2}<1 / 4$, the eigenvalue $\lambda= \pm i \kappa$ with $\kappa=\sqrt{1 / 4-k^{2}}$ in (A44). The first term in (A52) cannot meet the finite boundary condition when $\lambda=i \kappa$ unless it has the condition

$$
\frac{(\eta+1)}{2}-\frac{i \lambda}{2}=-n, \text { with } n=0,1,2,3, \cdots,
$$

which gives the quantization levels

$$
E=-\frac{\hbar^{2}}{2 M u_{0}^{2}}\left[\left(2 n+\left|n_{1}\right|+\frac{3}{2}\right)^{2}-\frac{1}{4}\right] .
$$

The parameter $\eta=(|\mu|+1 / 2)$ with $\mu=n_{1}$ was inserted to obtain the expression. When $\lambda=-i \mathcal{K}$, a normalizable solution requires $(\eta+1) / 2+i \lambda / 2=-n, n=0,1,2,3, \cdots$, which gives the same spectrum. The dynamic behavior of a particle on the $\mathrm{H}^{2}$ surface has been of interest to many of our colleagues over the decades (e.g., $[27,28])$. The conformal 
surface of $\mathrm{H}^{2}$ offers an additional degree of freedom, the conformal parameter $a$, to modulate the dynamic behavior of the particle, which can be achieved by the interaction with the external effective potential.

\section{References}

1. Leonhardt, U. Optical conformal mapping. Science 2006, 312, 1777-1780. [CrossRef] [PubMed]

2. Pendry, J.B.; Schurig, D.; Smith, D.R. Controlling electromagnetic fields. Science 2006, 312, 1780-1782. [CrossRef] [PubMed]

3. Chen, H.; Chan, C.T. Acoustic cloaking and transformation acoustics. J. Phys. D Appl. Phys. 2010, 43, 113001. [CrossRef]

4. Ni, X.; Wong, Z.-J.; Mrejen, M.; Wang, Y.; Zhang, X. An ultrathin invisibility skin cloak for visible light. Science 2015, 349, 1310-1313. [CrossRef] [PubMed]

5. Zhang, S.; Genov, D.A.; Sun, C.; Zhang, X. Cloaking of matter waves. Phys. Rev. Lett. 2008, 100, 123002-123004. [CrossRef] [PubMed]

6. Lin, D.-H. A mass-independent conformal quantum cloak. New J. Phys. 2017, 19, 113003. [CrossRef]

7. Lin, D.-H. Transformation design method for quantum states. Phys. Rev. A 2012, 85, 053605. [CrossRef]

8. Lin, D.-H. Form-invariant solution to quantum state on the sphere. J. Phys. Commun. 2020, 4, 085012. [CrossRef]

9. Weigold, E.; McCarthy, I.E. Electron Momentum Spectroscopy; Kluwer Academic: New York, NY, USA, 1999.

10. Lin, D.-H. Controlling matter waves in momentum space. Phys. Rev. A 2014, 90, 012709. [CrossRef]

11. Lin, D.-H. Quantum states emerging from charged transformation-wave in a uniform magnetic field. Eur. Phys. J. D 2016, 70, 141. [CrossRef]

12. Magnus, W.; Oberhettinger, F.; Soni, R.P. Formulas and Theorems for the Special Functions of Mathematical Physics; Springer: New York, NY, USA, 1966.

13. Newton, I. Philosophiae Naturalis Principia Mathematica; Cambridge University Press: Cambridge, UK, 1687.

14. Arnol'd, V.I. Huygens \& Barrow, Newton E Hooke; Birkhäuser Verlag: Basel, Switzerland, 1990.

15. Needham, T. Newton and the transmutation of force. Am. Math. Mon. 1993, 100, 119-137. [CrossRef]

16. Leonhardt, U.; Philbin, T. Geometry and Light: The Science of Invisibility; Dover: New York, NY, USA, 2010.

17. Lin, D.-H. The Hooke-newton transmutation system of magnetic force. J. Phys. Commun. 2018, 2, 065017. [CrossRef]

18. Lin, D.-H. On the magnetic Hooke-newton transmutation of electrons. J. Phys. Commun. 2019, 3, 085014. [CrossRef]

19. Morse, P.M.; Feshbach, H. Methods of Theoretical Physics; McGraw Hill: New York, NY, USA, 1953.

20. Lin, D.-H. On solutions of Coulomb system and its generalization to the Aharonov-Bohm effect. Ann. Phys. 2008, 323, 371-387. [CrossRef]

21. Nambu, Y.; Jona-Lasinio, G. Dynamical model of elementary particles based on an analogy with superconductivity. I. Phys. Rev. 1961, 122, 345. [CrossRef]

22. Goldstone, J.; Salam, A.; Weinberg, S. Broken symmetries. Phys. Rev. 1962, 127, 965. [CrossRef]

23. Gu, S.-J.; Yu, W.-C; Lin, H.-Q. Construct order parameters from the reduced density matrix spectra. Ann. Phys. 2013, 336, 118-129. [CrossRef]

24. Arraut, I. The Nambu-Goldstone theorem in nonrelativistic systems. Int. J. Mod. Phys. A 2017, 32, 1750127. [CrossRef]

25. Dane, C.; Verdiyev, Y.A. Integrable systems of group SO(1,2) and Green's functions. J. Math. Phys. 1996, 37, 39-60. [CrossRef]

26. Flügge, S. Practical Quantum Mechanics; Springer: Berlin/Heidelberg, Germany, 1971; Volume 2.

27. Gutzwiller, M.C. Chaos in Classical and Quantum Mechanics; Springer: Berlin/Heidelberg, Germany, 1990.

28. Grosche, C. Path Integrals, Hyperbolic Spaces and Selberg Trace Formulae; World Scientific: Singapore, 2013. 